\title{
O impacto da rizotomia dorsal seletiva na qualidade de vida de crianças espásticas portadoras de paralisia cerebral
}

\author{
José Aloysio Costa Val', Andréia Lara Oliveira Lima², Alexandra Oliveira Martins ${ }^{3}$
}

Serviço de Neurocirurgia Infantil do Biocor Instituto - Belo Horizonte, MG, Brasil

\section{RESUMO}

Objetivo: A rizotomia dorsal seletiva (RDS) é uma técnica clássica para tratamento da espasticidade em crianças com paralisia cerebral. Em nosso Serviço, é utilizada, além do objetivo funcional (deambulação), para melhoria da qualidade de vida da criança e do cuidador. O propósito do estudo foi avaliar a eficácia e o impacto do procedimento nesses indivíduos. Materiais e métodos: Um questionário sobre a qualidade de vida das crianças e cuidadores foi elaborado e enviado a 16 cuidadores de crianças submetidas à RDS, sendo posteriormente recuperados e analisados. Resultados: A maioria dos cuidadores julgou que houve melhora nos diversos quesitos relacionados à rotina diária, à facilitação para realização de cuidados e no posicionamento e transporte da criança. A maioria percebeu melhora no bem-estar e no estado de saúde. Dos cuidadores, $94 \%$ se declararam satisfeitos com o procedimento. Conclusão: $A$ $R D S$ parece melhorar a qualidade de vida de crianças gravemente comprometidas pela espasticidade na $P C$, bem como a de seus cuidadores.

\section{PALAVRAS-CHAVE}

Paralisia cerebral. Espasticidade. Rizotomia dorsal seletiva.

\begin{abstract}
The impact of selective dorsal rhizotomy on the quality of life of spastic children with cerebral palsy Objective: Selective dorsal rhizotomy (SDR) is a classical procedure for the treatment of spasticity in cerebral palsy children. In our Service the goal of this procedure is to improve quality of life, besides getting function (deambulation). In this study we analyze the effectiveness of the procedure. Methods: A questionnaire was send to 16 relatives that were caring children treated with SDR. Results: Almost all relatives considered improving in daily activities, facilitation to care, to transport and positioning the children, as well as improved health and welfare; $94 \%$ were satisfied with the procedure. Conclusion: SDR seems to improve quality of life of spastic children with cerebral palsy.
\end{abstract}

\section{KEY WORDS}

Cerebral palsy. Spasticity. Selective dorsal rhizotomy.

\section{Introdução}

A espasticidade é um sintoma comum em crianças com paralisia cerebral (PC). Em algumas condições, pode trazer benefício e, em outras, ser extremamente prejudicial e necessitar de tratamento. O tratamento da espasticidade é baseado em terapia física, medicamentos orais e procedimentos médicos invasivos. $\mathrm{O}$ tratamento cirúrgico é reservado para aquelas formas mais severas ou generalizadas de espasticidade, com sintomas muito intensos. Infelizmente, estas são situações comuns na $\mathrm{PC}^{21}$. $\mathrm{O}$ tratamento neurocirúrgico da espasticidade complementa e auxilia as diversas modalidades terapêuticas existentes. Até 1987, quando se difundiu a rizotomia dorsal seletiva $(\mathrm{RDS})^{1}$, o neurocirurgião pediátrico pouco contato tinha com essas crianças. Com o advento dessa técnica, a atenção desses profissionais foi atraída para o problema e mais técnicas foram desenvolvidas e realizadas. A aplicação da toxina botulínica intramuscular, a própria RDS e a

1 Neurocirurgião pediátrico e coordenador do Serviço de Neurocirurgia Infantil do Biocor Instituto. Belo Horizonte, MG.

2 Neuropediatra do Biocor Instituto. Belo Horizonte, MG.

3 Psicóloga hospitalar infantil do Biocor Instituto. Belo Horizonte, MG. 
administração intratecal de baclofeno são hoje técnicas muito úteis no manejo da espasticidade $e^{2-4,8,16-18,25}$.

A RDS é uma técnica segura e realizável na maioria dos centros capacitados a exercer a neurocirurgia infantil, demandando pouca tecnologia e com custo acessível. A partir de 2001, tornou-se o procedimento de escolha para tratamento de crianças quadriespásticas no Serviço de Neurocirurgia Infantil do Biocor Instituto. Tem como objetivos a melhora funcional - infelizmente obtida na minoria das crianças - e a melhora de qualidade de vida, no conforto e nos cuidados ${ }^{7}$.

A fim de compreender os efeitos da RDS na qualidade de vida dessas crianças e seus cuidadores, propôs-se a realização do estudo a seguir.

\section{Técnica e objetivos}

A RDS é uma cirurgia convencional realizada sob anestesia geral. A cauda eqüina é exposta por laminotomia osteoplástica lombar, sendo identificadas as raízes dorsais sensitivas de $\mathrm{S} 1$ a $\mathrm{L} 2$ bilateralmente, confirmadas após estímulos neurais. Uma lesão compreendendo cerca de $30 \%$ de seu diâmetro é realizada em cada raiz.

O efeito alcançado é, em geral, a diminuição de dois níveis na escala de tônus de Ashorth no conjunto muscular espástico dos membros inferiores. Em cerca de $50 \%$ das crianças, esse efeito estende-se também aos membros superiores.

Os objetivos do procedimento, sempre realizado após discussão em equipe multidisciplinar, são de melhoria funcional (quando possível) e melhora na qualidade de vida ${ }^{7}$.

Como melhoria de qualidade de vida, diversos fatores são considerados:

- conforto físico da criança (diminuição da dor, melhoria no posicionamento, facilitar ou permitir higienes genital, axilar e cervical);

- melhoria nas funções do cuidador (transporte, posicionamento, higiene);

- contribuição para profilaxia de complicações físicas (escaras, luxações, escoliose, deformidades).

\section{Casuística e métodos}

Estudaram-se, retrospectivamente, 25 crianças submetidas à RDS entre março de 2001 e fevereiro de 2005. Deste grupo inicial, três crianças já haviam falecido de outras causas e seis não foram localizadas.
Um questionário sobre a qualidade de vida das crianças e dos cuidadores foi elaborado, baseado no questionário da World Health Organization Quality of Life (WHOQOL), do Programa de Saúde Mental da $\mathrm{OMS}^{26}$ e em experiência local da equipe.

Esse questionário foi enviado a 16 cuidadores (15 mães e uma avó), informando-se também os objetivos do trabalho e solicitando-se autorização para o uso dos dados. Após preenchimento e obtendo a autorização, os questionários foram recuperados e os dados, analisados por uma neuropediatra e uma psicóloga infantil.

\section{Resultados}

A idade de realização do procedimento variou de 3 a 15 anos. A maioria situou-se entre os 5 e 8 anos.

A etiologia predominante da $\mathrm{PC}$ foi hipóxia perinatal, presente em $46 \%$ dos casos. Infecções pós-natais foram a causa em $20 \%$, hemorragia ventricular do recém-nascido, em $21 \%$ e kernicterus, em $13 \%$.

Doze crianças $(75 \%)$ apresentaram grave comprometimento cognitivo e, em quatro, o comprometimento foi moderado.

O comprometimento motor mensurado segundo o GMFCS (Gross Motor Function Classification System) ${ }^{19}$ atingiu grau $\mathrm{V}$ em 13 pacientes e grau III em 3.

A análise do impacto da RDS nos cuidados diários está apresentada na tabela 1 .

Tabela 1

Grau de dificuldade na realização dos cuidados cotidianos antes e depois da RDS (número de crianças)

\begin{tabular}{lcccc}
\hline Cuidados & \multicolumn{2}{c}{ Antes da RDS } & \multicolumn{2}{c}{ Depois da RDS } \\
\cline { 2 - 5 } & $\begin{array}{c}\text { Fácil ou } \\
\text { moderado }\end{array}$ & $\begin{array}{c}\text { Muito difícil } \\
\text { ou difícil }\end{array}$ & $\begin{array}{c}\text { Fácil ou } \\
\text { moderado }\end{array}$ & $\begin{array}{c}\text { Muito difícil } \\
\text { ou difícil }\end{array}$ \\
\hline Alimentação & 7 & 9 & 14 & 2 \\
Banho & 4 & 12 & 13 & 3 \\
Higiene & 3 & 13 & 13 & 3 \\
Troca de frauda & 3 & 13 & 12 & 4 \\
Troca de roupa & 3 & 13 & 12 & 4 \\
\hline
\end{tabular}

A análise das respostas à questão específica "quanto melhorou em todos os cuidados após a cirurgia?” está apresentada na figura 1.

Em relação ao impacto da RDS no posicionamento e transporte, os resultados estão apresentados na tabela 2 .

A análise das respostas à questão específica "após a cirurgia quanto melhorou para posicionar e transportar a criança?" está apresentada na figura 2.

Em relação à percepção do bem-estar e saúde da criança e o impacto da RDS, os resultados são apresentados na tabela 3 . 

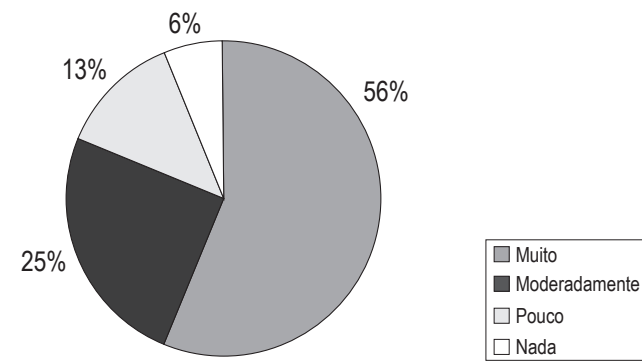

Figura 1-Análise das respostas à questão "quanto melhorou em todos os cuidados após a cirurgia?".

Tabela 2

Impacto no posicionamento e transporte (número de crianças)

\begin{tabular}{lcccc}
\hline Posicionamento $\begin{array}{c}\text { Antes da RDS } \\
\text { transporte }\end{array}$ & $\begin{array}{c}\text { Fácil ou } \\
\text { moderado }\end{array}$ & $\begin{array}{c}\text { Difícil ou } \\
\text { muito difícil }\end{array}$ & $\begin{array}{c}\text { Fácil ou } \\
\text { moderado }\end{array}$ & $\begin{array}{c}\text { Difícil ou } \\
\text { muito difícil }\end{array}$
\end{tabular}

\begin{tabular}{lllll}
\hline Posicionar & 3 & 13 & 10 & 6 \\
Transportar & 3 & 13 & 11 & 5 \\
Carregar no colo & 3 & 13 & 11 & 5 \\
Assentar & 4 & 12 & 15 & 1 \\
\hline
\end{tabular}
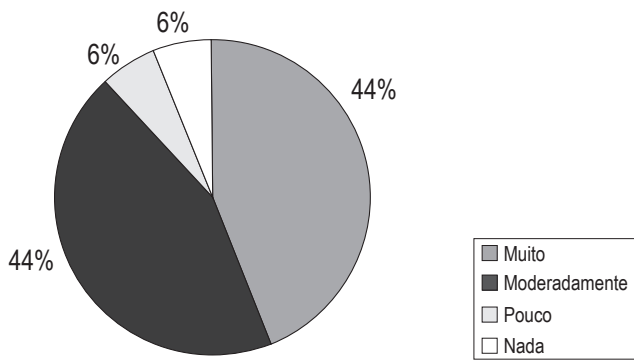

Figura 2-Análise das respostas à questão "após a cirurgia quanto melhorou para posicionar e transportar a criança?".

Tabela 3

Impacto no bem-estar e saúde da criança (número de crianças)

\begin{tabular}{lcccc}
\hline \multirow{2}{*}{$\begin{array}{l}\text { Bem-estar } \\
\text { e saúde }\end{array}$} & \multicolumn{2}{c}{ Antes da RDS } & \multicolumn{2}{c}{ Depois da RDS } \\
\cline { 2 - 5 } & $\begin{array}{c}\text { Raramente } \\
\text { ou nunca }\end{array}$ & $\begin{array}{c}\text { Sempre ou } \\
\text { quase sempre }\end{array}$ & $\begin{array}{c}\text { Raramente } \\
\text { ou nunca }\end{array}$ & $\begin{array}{c}\text { Sempre ou } \\
\text { quase sempre }\end{array}$
\end{tabular}

\begin{tabular}{lcccc}
\hline $\begin{array}{l}\text { Bom sono } \\
\begin{array}{l}\text { Ganho } \\
\text { de peso }\end{array}\end{array}$ & 4 & 12 & 2 & 14 \\
$\begin{array}{l}\text { Uso de } \\
\text { analgésico }\end{array}$ & 13 & 3 & 13 & 3 \\
$\begin{array}{l}\text { Dor } \\
\text { espontânea }\end{array}$ & 7 & 3 & 13 & 3 \\
$\begin{array}{l}\text { Dor à } \\
\text { manipulação }\end{array}$ & 5 & 8 & 14 & 2 \\
\hline
\end{tabular}

A análise das respostas à questão específica "quanto melhorou o estado de saúde e o bem-estar da criança após a cirurgia?" está apresentada na figura 3.

$\mathrm{O}$ grau de satisfação com a cirurgia foi inquirido pela questão "quão satisfeito você está com a cirurgia?" e as respostas constam na figura 4 .

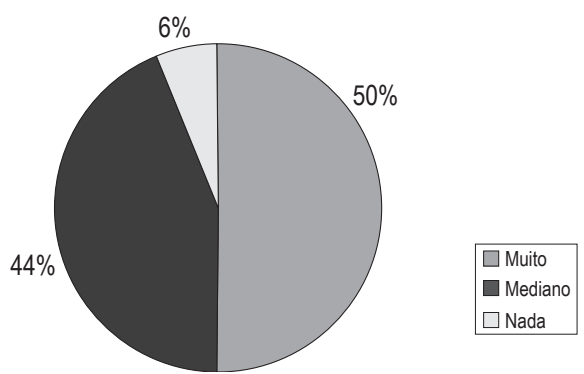

Figura 3 - Percepção da melhora do estado de saúde e bem-estar da criança.

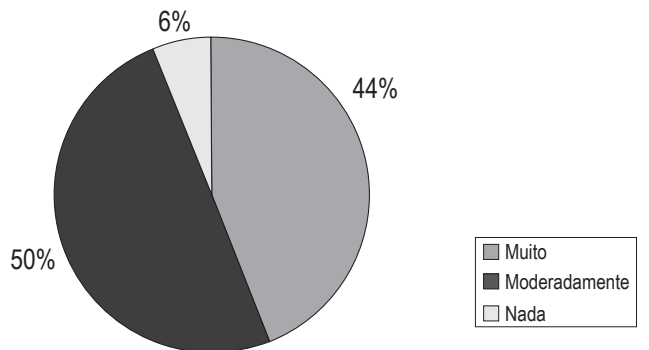

Figura 4-Grau de satisfação com a cirurgia.

Por fim, analisaram-se as questões direcionadas à qualidade de vida do cuidador, baseadas no questionário World Health Organization Quality of Life $(\mathrm{WHOQOL})^{26}$. A maioria relatou boa qualidade de vida: $80 \%$ relataram aproveitar a vida muito ou medianamente, $90 \%$ relataram muita ou mediana energia para o dia a dia, $80 \%$ relataram que a vida valia a pena e $80 \%$ qualificaram sua vida como boa ou muito boa.

\section{Discussão}

A incidência da paralisia cerebral vem aumentando em relação direta com a melhoria dos cuidados neonatais, que permitem a sobrevida de crianças anteriormente inviáveis. Essas crianças têm uma chance muito maior de desenvolver a lesão $0^{5,23}$.

A espasticidade é uma comorbidade presente na maioria dessas crianças. A ação constante da hipertonia causa manifestações funcionais, sobretudo na marcha, 
bem como deformidades esqueléticas. Um tratamento agressivo e precoce, baseado em terapia física, reabilitação e procedimentos médicos, melhora a funcionalidade dessas crianças.

Alguns pacientes, entretanto, têm uma qualidade de vida muito ruim em razão da dor, dificuldade de higiene, mobilização e transporte. Esses problemas são potencializados em crianças com a função cognitiva comprometida que, entre vários outros fatores, não podem expressar-se.

A RDS é uma técnica clássica, com origem no século XIX e desenvolvida durante o século $\mathrm{XX}^{9,10,19,22}$. Atualmente é utilizada, na maioria dos centros especializados, para tratamento da espasticidade, com o objetivo de melhorar a função. É o tratamento de escolha para crianças diparéticas espásticas que não deambulam, mas têm força preservada ${ }^{2,20,24}$. Há, também, evidências de impacto positivo da técnica em procedimentos médicos e de reabilitação posteriores ${ }^{6,11,12,15,20}$.

Em nosso Serviço, há predomínio de crianças muito comprometidas cognitivamente, sem expectativa de função motora. $\mathrm{O}$ tratamento indicado nesses casos visa a facilitar cuidados, melhorar a qualidade de vida da criança e de seus cuidadores. Neste contexto, a melhor técnica é a utilização do baclofeno intratecal ${ }^{3,8,16}$. Infelizmente essa técnica não está disponível para a maioria dos pacientes. Indica-se, na literatura, o uso da RDS para propiciar cuidados e melhoria da qualidade de vida em crianças muito comprometidas cognitivamente ${ }^{2,3,13,14}$. Esta, também, é a prática em nosso Serviço.

A observação dos resultados obtidos na entrevista de 16 cuidadores de crianças com espasticidade submetidas à RDS mostrou dados interessantes. Praticamente em todas elas o objetivo não foi primariamente funcional, e, sim, facilitar os cuidados. A realização das atividades primárias do cotidiano (alimentação, banho, higiene, troca de frauda e roupa) era difícil para a maioria das crianças. O procedimento parece ter propiciado uma inversão neste quadro. Mais de $80 \%$ dos cuidadores consideraram a realização dessas tarefas mais fáceis após a RDS.

Os maiores problemas encontrados pelos cuidadores são o posicionamento e transporte. Uma postura inadequada acentua todas as manifestações de espasticidade, além de contribuir para o aparecimento de deformidades, contraturas e escaras. À medida que o paciente cresce, o transporte, na vigência da espasticidade, pode tornar-se quase impossível. A análise das respostas sugere que os atos de posicionar, transportar, carregar no colo e assentar tornaram-se mais fáceis para a maioria, após a RDS. Para $88 \%$ dos cuidadores, houve melhora nesses itens.

Em relação ao bem-estar e saúde, parece ter ocorrido melhoria quanto à percepção de dor espontânea ou à manipulação da criança. Quanto à qualidade do sono, ao uso de analgésicos e ao ganho de peso, não houve impacto significativo. Entretanto, para $94 \%$ dos cuidadores, houve percepção de melhora da qualidade de vida para a criança.

Novamente, $94 \%$ desses cuidadores afirmaram estar satisfeitos com a cirurgia.

De acordo com esses dados, sugere-se que a RDS consegue interferir na qualidade de vida dessas crianças de maneira positiva.

\section{Conclusão}

A paralisia cerebral é uma manifestação cada vez mais incidente em todo o mundo, em razão da melhora dos cuidados neonatais. A espasticidade ocorre na maioria dessas crianças e causa, além de problemas funcionais e deformidades, dificuldade para realização de cuidados básicos, dor e qualidade de vida ruim para os pacientes e seus cuidadores.

A RDS, utilizada como alternativa para o tratamento da espasticidade com a proposta de melhorar a qualidade de vida, parece atingir esse objetivo, influindo positivamente na vida das crianças e seus cuidadores.

\section{Referências}

1. ABBOTT R, JOHANN-MURPHY M, SHIMINSKY-MAHER $T$ et al.: Selective dorsal rhisotomy: outcome and complications in treating spastic cerebral palsy. Neurosurgery 33:851-7, 1993.

2. ALBRIGTH AL: Spasticity and movement disorders. In Albrigth AL, Pollack I, Adelson D (eds): Principles and practice of pediatric neurosurgery. New York, Thieme Medical Publishers, 1999.

3. ALBRIGTH AL, BARRON WB, FAICK MP et al.: Continuos intrathecal baclofen infusion for spasticity of cerebral origin. JAMA 270:2475-7, 1993.

4. AMSTRONG RW, STENIBOK P, COCHRANE DD et al.: Intratecally administered baclofen for treatment of children with spasticity of cerebral origin. J Neurosurg 87:409-14, 1997.

5. CAHAN LD, ADAMS JM, PERRY J, BEELER LM: Instrumented gait analysis after selective dorsal rhisotomy. Dev Med Child Neurol 32:1037-43, 1990.

6. CHICOINE MR, PARK TS, KAUFMANN BA: Selective dorsal rhisotomy and rates of orthopedic surgery in children with cerebral palsy. J Neurosurg 86:34-9, 1997.

7. COSTAVAL FILHO JA: Manejo da espasticidade na paralisia cerebral. In Lima C, Fonseca LF (eds): Paralisia cerebral. Rio de Janeiro, Medsi, 2004, pp 119-27.

8. DRALLE D, MULLER H, ZIERSK J, KLUG N: Intrathecal baclofen for spasticity. Lancet 2:1003, 1985. 
9. FASANO VA, BROGGI G, BAROLAT- ROMANA GB, SGUAZZI A: Surgical treatment of spasticity in cerebral palsy. Childs Brain 4:289-305, 1978.

10. FOESTER O: On the indications and results of the excision of posterior nerve roots in men. Surg Gynecol Obstet 16:463-74, 1913.

11. GUL SM, STEINBOK P, MCLEOD K: Long-term outcome after selective posterior rhizotomy in children with spastic cerebral palsy. Ped Neurosurg 31:84-95, 1999.

12. HICDONMEZ T, STEINBOK P, BEAUCHAMP R, SAWATZKY B: Hip joint subluxation after selective dorsal rhizotomy for spastic cerebral palsy. J Neurosurg (Pediatrics 1) $103: 10-16,2005$.

13. ILLUM NO, TORP-PEDERSEN L, MIDHOLM S, SELMAR PE, SIMESEN K Rhizotomy for children with severe spastic cerebral palsy. Ugeskr Laeger 168:785-9, 2006.

14. KIM DS, CUOI JU, YANG KH, PARK Cl: Selective posterior rhisotomy in chidren with cerebal palsy: a 10-year experience. Child's Nerv Syst 17:556-62, 2001.

15. KIM HS, STEINBOK P, WICKENHEISER D: Predictors of poor outcome after selective dorsal rhizotomy in treatment of spastic cerebral palsy. Childs Nerv Syst 22:60-6, 2006.

16. KNUTSSON E, LINDBLOM U, MATERSON A: Plasma and cerebrospinal fluid levels of baclofen (Lioresal) at optimal responses in spastic paraparesis. J Neurol Sci 23: 473-484, 1974.

17. MULLER H: Treatment of severe spasticity: results of a multicenter trial conducted in Germany involving the intrathecal infusion of baclofen by an implantable drug delivery system. Dev Med Child Neurol 34:739-45, 1992.

18. MULLER H, ZIERSKI J, DRALLE D et al.: Pharmacokinectics of intrathecal baclofen. In Muller H, Zierski J, Penn RD (eds): Local spinal therapy for spasticity. New York, Springer-Verlag, 1988, pp 223-6.
19. PALISASO RJ, HANNA SE, ROSENBAUM PL, RUSSEL DJ, WALKER SD, WOOD EP et al.: Validation of a model of Gross motor function for children with cerebral palsy. Phys Ther 80:974-85, 2000

20. PARK TS, VOGLER GP, PHILIPS LH et al.: Effects of selective dorsal rhisotomy for spastic diplegia on hip migration in cerebral palsy. Pediatric Neurosurg 20:43-9, 1994.

21. PEACOCK WJ, ARENS LJ, BERMAM B: Cerebral palsy spasticity. Selective posterior rhizotomy. Pediatric Neurosci 13:61-6, 1987.

22. SHERRINGTON C: Decerebrate rigidity and reflex coordination of movements. J Physiol (London) 22:319-27, 1898.

23. STANLEY FJ: Survival and cerebral palsy in low birth weight infants: implications for perinatal care. Paediatric Perinat Epidemiol 6:298-310, 1992.

24. STEINBOK P: Outcomes after selective dorsal rhizotomy for spastic cerebral palsy. Child's Nerv Syst 17:1-18, 2001.

25. STEINBOK P: Neurosurgical management of hypertonia in children. Neurosurg Quartely 12:63-78, 2002.

26. WORD HEALTH ORGANIZATION: Portuguese version of the instrument for the assessment of quality of life of the World Health Organization (WHOQOL-100).

Original recebido em junho de 2007

Aprovado para publicação em janeiro de 2008

\section{Endereço para correspondência}

José Aloysio da Costa Val

Av. Alameda da Serra, 322/sala 408

34000-000 - Nova Lima, $M G$

E-mail:costaval.bh@terra.com.br 